ROCKY MOUNTAIN

JOURNAL OF MATHEMATICS

Volume 46, Number 6, 2016

\title{
VOLUME INDEX
}

Abdallah, Ahmed Y., Asymptotic behavior of strongly damped nonlinear beam equations, 1071.

Abd-Elhameed, W.M., see Doha, E.H.

Abdolghafourian, Adeleh, On the divisibility graph for finite sets of positive integers, 1755 .

Altıntaş Sharland, A., Nonisolated forms of rational triple point singularities of surfaces and their resolutions, 357.

Amini, Massoud, $\mathrm{C}^{*}$-algebras of 2-groupoids, 693.

Ansari-Toroghy, H., On the graph of modules over commutative rings, 729 .

Arizmendi-Peimbert, Hugo, On algebras of Banach algebra-valued bounded continuous functions, 389 .

Bakherad, Mojtaba, Reverse Young-type inequalities for matrices and operators, 1089.

Baragar, Arthur, Automorphisms of surfaces in a class of Wehler K3 surfaces with Picard number 4, 399.

Barreira, Luis, Exponential dichotomies for impulsive equations via quadratic functions, 1771.

Bates, R., Quadratic hyperbolicity preservers and multiplier sequences, 51.

Beauregard, Raymond A., A discrete view of Faà di Bruno, 73.

Beltiţă, Daniel, see Beltiţă, Ingrid.

Beltiţă, Ingrid, Symbol calculus of square-integrable operator-valued maps, 1795.

Benjamin, Elliot, Some fifth roots that are constructible by marked ruler and compass, 749 .

Beuter, Viviane M., Partial crossed products as equivalence relation algebras, 85 .

Bonan-Hamada, C., Survey Article: Continued fractions associated with WienerLevinson filters, frequency analysis, moment theory and polynomials orthogonal on the unit circle, 1.

Boua, A., see Samman, M.

Brown, Jim, Amicable pairs and aliquot cycles for elliptic curves over number fields, 1853.

Cao, Xiaodong, On the mean of the shifted error term in the theory of the Dirichlet divisor problem, 105.

Caprau, Carmen, Singular links and Yang-Baxter state models, 1867.

Carrillo-Hoyo, Angel, see Arizmendi-Peimbert, Hugo.

Casnati, Gianfranco, On the rationality of Poincaré series of Gorenstein algebras via Macaulay's correspondence, 413.

Çelik, Mehmet, Nilpotent Toeplitz operators on Reinhardt domains, 1395.

Çevik, G., see Altıntaş Sharland, A.

Chebolu, Sunil, Galois p-groups and Galois modules, 1405.

Chen, Bohui, see Du, Song.

Chen, Youhua, see Yin, Huayu.

Choudhry, Ajai, Quadratic diophantine equations with applications to quartic equations, 769 .

Chu, Y.M., see Wang, M.K.

Cortes, Wagner, Partial crossed products and fully weakly prime rings, 1107.

DeBlois, Jason, The geometry of cyclic hyperbolic polygons, 801.

Denker, Manfred, see Koslicki, David.

Deshpande, Priyavrat, Arrangements of spheres and projective spaces, 1447.

DOI:10.1216/RMJ-2016-46-6-2089 Copyright (C)2016 Rocky Mountain Mathematics Consortium 
Diaz-Vargas, Javier, Riemann hypothesis for the Goss t-adic zeta function, 435.

Dobrushkin, Vladimir A., see Beauregard, Raymond A.

Doha, E.H., New linearization formulae for the products of Chebyshev polynomials of third and fourth kinds, 443.

Dong Quan, Nguyen Ngoc, Non-vanishing of Carlitz-Fermat quotients modulo primes, 125 .

Dorff, Michael, see Kumar, Raj.

Du, Cheng-Yong, see Du Song.

$\mathrm{Du}$, Song, Ruan cohomologies of the compactifications of resolved orbifold conifolds, 863.

Duncan, Benton L., Graph theoretic invariants for operator algebras associated to topological dynamics, 131.

Eftekharinasab, Kaveh, Geometry of bounded Fréchet manifolds, 895.

Faucett, Jessica Ann, Expanding the socle of a codimension 3 complete intersection, 1489.

Feng, Yibin, General mixed chord-integrals of star bodies, 1499.

Furuya, Jun, see Cao, Xiaodong.

Gao, Xiang, Characterizations of the compactness of Riemannian manifolds by eigenfunctions, and a partial proof of a conjecture by Hamilton, 461.

Garbagnati, Alice, Kummer surfaces and $\mathrm{K} 3$ surfaces with $(\mathbb{Z} / 2 \mathbb{Z})^{4}$ symplectic action, 1141.

García-García, Alejandra, see Arizmendi-Peimbert, Hugo.

Gillaspy, Elizabeth, K-theory and homotopies of 2-cocycles on group bundles, 1207.

de Godoi, Juliano D.B., A class of nonlinear elliptic systems with Steklov-Neumann nonlinear boundary conditions, 1519.

Goldin, Rebecca, Inertia groups of a toric Deligne-Mumford stack, fake weighted projective stacks, and labeled sheared simplices, 481.

Gonçalves, Daniel, see Beuter, Viviane M.

González-Jiménez, Enrique, Torsion of rational elliptic curves over cubic fields, 1899.

Grigorian, G.A., Some properties of the solutions of third order linear ordinary differential equations, 147.

Grigoryan, Armen, Slit univalent harmonic mappings, 169.

Guo, Bai-Ni, An explicit formula for Bernoulli polynomials in terms of $\mathbf{r}$-Stirling numbers of the second kind, 1919.

Guo, Peng, Smooth center manifolds for random dynamical systems, 1925.

Gupta, Sushma, see Kumar, Raj.

Habibi, Sh., see Ansari-Toroghy, H.

Harada, Megumi, see Goldin, Rebecca.

Hayasaka, Futoshi, A computation of Buchsbaum-Rim functions of two variables in a special case, 1547.

Heras, David, see Brown, Jim.

Honari, B., see Kalantari, Sh.

$\mathrm{Hu}$, Shuna, see Li, Shuchao.

Iranmanesh, Mohammad A., see Abdolghafourian, Adeleh.

James, Kevin, see Brown, Jim.

James, Rodney, Linear systems on edge-weighted graphs, 1559.

Jefferies, Brian, Bilinear integration and applications to operator and scattering theory, 189.

Jelisiejew, Joachim, see Casnati, Gianfranco.

Ji, Guanghua, Lower bound for the higher moment of symmetric square L-functions, 915. 
Jiang, Y.P., see Wang, M.K.

Johannsen, David, see Goldin, Rebecca.

Jones, W.B., see Bonan-Hamada, C.

Kalantari, Sh., Asymptotic resemblance, 1231.

Kara, Yeliz, Modules whose certain submodules are essentially embedded in direct summands, 519 .

Kaygisiz, Kenan, Determinantal and permanental representations of Fibonacci type numbers and polynomials, 227.

Keaton, Rodney, see Brown, Jim.

Koslicki, David, Substitution Markov chains and Martin boundaries, 1963.

Krantz, Steven G., The Greene-Krantz conjecture in dimension two, 1575.

Krespki, Derek, see Goldin, Rebecca.

Krnić, Mario, see Bakherad, Mojtaba.

Kuhlmann, Katarzyna, The structure of spaces of $\mathbb{R}$-places of rational function fields over real closed fields, 533.

Kumar, Raj, An application of Cohn's rule to convolutions of univalent harmonic mappings, 559.

Kwon, Bo-Hyun, On the HOMFLY polynomial of 4-plat presentations of knots, 243.

Lalín, Matilde, The number of irreducible polynomials with the first two prescribed coefficients over a finite field, 1587.

Larocque, Olivier, see Lalín, Matilde.

Lee, Jae-Hyouk, Contractions of del Pezzo surfaces to $\mathbb{P}^{2}$ or $\mathbb{P}^{1} \times \mathbb{P}^{1}, 1263$.

Lee, Juhyung, A functional equation and degenerate principal series, 1987.

Léka, Zoltán, A note on extremal decompositions of covariances, 571.

$\mathrm{Li}$, Hong-Hai, The minimum matching energy of bicyclic graphs with given girth, 1275 .

$\mathrm{Li}$, Shuchao, On the spectral moment of graphs with given clique number, 261.

$\mathrm{Li}$, Xiaobin, see Du, Song.

Li, Yong, see Wang, Chuanbiao.

Liang, Yongqi, Progress concerning the local-global principle for zero-cycles on algebraic varieties, 1293.

Liu, Xia, see Shi, Haiping.

Liu, Zhongkui, see Ma, Xin.

Llibre, Jaume, Polynomial first integrals for weight-homogeneous planar polynomial differential systems of weight degree 4, 1619.

Ma, Xin, Strongly copure projective, injective and flat complexes, 2017.

Măntoiu, Marius, see Beltiţă, Ingrid.

Manzoli, Donald J., Ramanujan: A tale of two evaluations, 925.

Mayes, Sarah, The symbolic generic initial system of almost linear point configurations in $\mathbb{P}^{2}, 283$.

Mező, István, see Guo, Bai-Ni.

Mináč, Ján, see Chebolu, Sunil.

Miranda, Rick, see James, Rodney.

Miyagaki, Olímpio H., see de Godoi, Juliano D.B.

Mooney, Christopher Park, $\tau$-Regular factorization in commutative rings with zerodivisors, 1309.

Moslehian, Mohammad Sal, see Bakherad, Mojtaba.

Moslehian, Mohammad Sal, see Sadeghi, Ghadir.

Moya-Pérez, J.A., Topological triviality of families of map germs from $\mathbb{R}^{3}$ to $\mathbb{R}^{3}$, 1643.

Mphako-Banda, Eunice, Graph compositions of suspended $Y$-trees, 1351.

Munagi, Augustine O., On singletons and adjacencies of set partitions, 301. 
Najman, Filip, see González-Jiménez, Enrique.

Navarro, Gabriel, Variations on the Itô-Michler theorem on character degrees, 1363.

Nielsen, Morten, On Schauder basis properties of multiply generated Gabor systems, 2043.

Njåstad, O., see Bonan-Hamada, C.

Notari, Roberto, see Casnati, Gianfranco.

Nuño-Ballesteros, J.J., see Moya-Pérez, J.A.

Okano, Tsutomu, see Caprau, Carmen.

Orton, Danny, see Caprau, Carmen.

Orum, Chris, Golomb's arithmetical semigroup topology and a semi-prime suffciency condition for Dirichlet's theorem, 939.

Oukhtite, L., see Samman, M.

Pérez, Marco A., Homological dimensions and Abelian model structures on chain complexes, 951.

Pimentel, Edgard A., Estimates for a class of slowly non-dissipative reactiondiffusion equations, 1011.

Pimentel, Juliana F.S., see Pimentel, Edgard A.

Polanco-Chi, Enrique, see Diaz-Vargas, Javier.

Previte, Joseph, Copy-paste trees and their growth rates, 1029.

Previte, Michelle, see Previte, Joseph.

Qi, Feng, see Guo, Bai-Ni.

Qian, Andrew, see Brown, Jim.

Raji, A., see Samman, M.

Rau, Johannes, Intersections on tropical moduli spaces, 581.

Rodrigues, Rodrigo S., see de Godoi, Juliano D.B.

Sadeghi, Ghadir, Inequalities for sums of random variables in noncommutative probability spaces, 309.

Şahin, Adem, see Kaygisiz, Kenan.

Salem, Ahmed, A certain class of approximations for the q-digamma function, 1665.

Salgado, Cecília, Arithmetic and geometry of rational elliptic surfaces, 2061.

Sal Moslehian, Mohammad, see Bakherad, Mojtaba.

Samman, M., Two sided $\alpha$-derivations in 3-prime near-rings, 1379.

Sarti, Alessandra, see Garbagnati, Alice.

Schultz, Andrew, see Chebolu, Sunil.

Shen, Jun, see Guo, Peng.

Shi, Haiping, Existence of periodic solutions for 2 thth-order nonlinear p-Laplacian difference equations, 1679.

Singh, Sukhjit, see Kumar, Raj.

Snyder, C., see Benjamin, Elliot.

Soares, Marlon, see Cortes, Wagner.

Stopple, Jeffrey, Notes on $\log (\zeta(s))^{\prime \prime}, 1701$.

Szirmai, Jenő, Non-periodic geodesic ball packings generated by infinite regular prism tilings in $\widetilde{\mathbf{S L}_{2} \mathbf{R}}$ space, 1055.

Tang, Mingtian, see Wang, Yunyan.

Tanigawa, Yoshio, see Cao, Xiaodong.

Tercan, Adnan, see Kara, Yeliz.

Tornero, José M., see González-Jiménez, Enrique.

Tosun, M., see Altıntaş Sharland, A.

Trudgian, Tim, Improvements to Turing's method II, 325.

Valls, Claudia, see Barreira, Luis. 
Valls, Claudia, see Llibre, Jaume.

Vanderschoot, Mary, see Previte, Joseph.

Wang, Bin, First order deformations of pairs and non-existence of rational curves, 663.

Wang, Chuanbiao, Affine-periodic solutions for nonlinear differential equations, 1717.

Wang, M.K., Ramanujan's cubic transformation inequalities for zero-balanced hypergeometric functions, 679.

Wang, Weidong, see Feng, Yibin.

Wang, Yunyan, Local $M$-estimation for conditional variance function with dependent data, 333.

Werner, Simon, see Mphako-Banda, Eunice.

Yan, Xiaoguang, see Zhao, Guoqiang.

Yang, Xue, see Wang, Chuanbiao.

Yin, Huayu, Content formulas for power series and Krull domains, 2077.

Yoshida, R., see Bates, R.

Zeytuncu, Yunus E., see Çelik, Mehmet.

Zhai, Wenguang, see Cao, Xiaodong.

Zhang, Yuanbiao, see Shi, Haiping.

Zhao, Guoqiang, Resolutions and stability of C-Gorenstein flat modules, 1739.

Zhu, Xiaosheng, see Yin, Huayu.

Zou, Li, see Li, Hong-Hai. 